\title{
Particle Imaging Velocimetry Measurement of Air Flow Between High Voltage Asymmetrical Electrodes
}

\author{
Jiř́ Primas ${ }^{1}$, Michal Malík ${ }^{1, *}$, Petr Schovanec ${ }^{1}$, Josef Novák $^{1}$, Pavel Pokorný ${ }^{2}$, and Filip \\ Sanetrník ${ }^{2}$ \\ ${ }^{1}$ Technical university of Liberec, Faculty of mechatronics, Hálkova 6, Liberec, 461 17, Czech \\ Republic \\ ${ }^{2}$ Technical university of Liberec, Faculty of textile engineering, Studentská 2, Liberec, 461 17, Czech \\ Republic
}

\begin{abstract}
This paper deals with the airflow generation phenomenon occurring on a system of strongly asymmetrical electrodes connected to high DC voltage. The main focus was to measure the airflow directly between the electrodes using Particle Image Velocimetry. The authors are well aware of the many difficulties presented by the task of measuring in the presence of strong electric fields and the paper also describes the means used to overcome these issues. The results of performed measurements are presented, their agreement with theoretical description of given phenomenon is discussed and several possible practical applications are proposed.
\end{abstract}

\section{Introduction}

It is a well-known phenomenon that on a system of strongly asymmetrical electrodes connected to high DC voltage (in the range of tens of kilovolts) a flow of surrounding air will occur $[1,2]$. To describe the exact flow field and to measure the precise magnitudes of velocity vectors is a basic task in experimental fluid mechanics. For this purpose there are many well-described and routine methods - e.g. Laser Doppler Anemometry (LDA), sophisticated Constant Temperature Anemometry (CTA) measuring systems or Particle Imaging Velocimetry (PIV) $[3,4,5]$. In this article we are going to describe the obstacles and specific problems and their solutions of measuring the flow in the presence of high DC voltage using the PIV method. This method was chosen because of its following advantages. It is a precise contactless measuring method with minimal invasiveness and with flexible measuring range including the velocities we expect in our experiment. Last but not least it is also available at Technical university of Liberec and our laboratory has a long-time practical experience with it $[6,7]$.

The basic principle of measuring liquid or gas flow velocity using PIV method is based on registering the shift of seeding particles, which must follow the measured flow. This is done using sets of two consecutive planar laser light pulses, while a digital camera records

${ }^{*}$ Corresponding author: michal.malik@tul.cz 
corresponding images of the seed particles position with small time delay between them. Recorded images are further processed and analysed by specialized software to extract the information about the flow field velocities in the measuring plane.

\section{Experiment}

First of all the system of asymmetrical electrodes was constructed. It consists of a rectangular conductive aluminium box with measures $100 \times 50 \times 10 \mathrm{~mm}$ acting as a larger grounded electrode and of thin copper wire with radius $0.04 \mathrm{~mm}$ stretched between glass insulating rods parallel to the larger electrode in $30 \mathrm{~mm}$ distance, see Figure 1. This wire acts as a smaller electrode connected to high DC voltage.

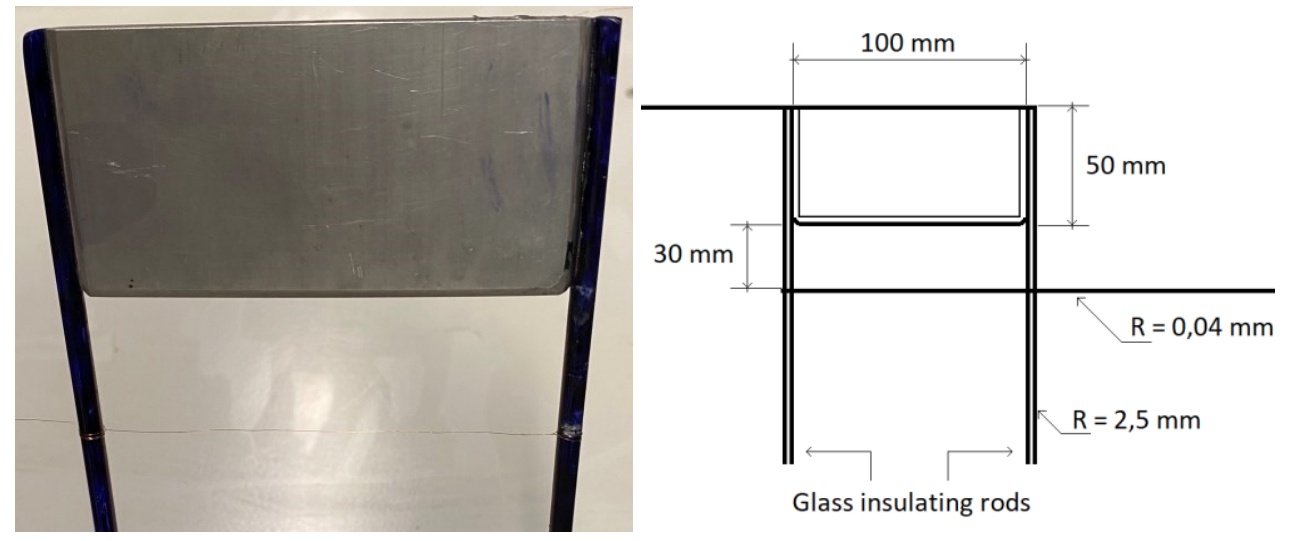

Fig. 1. Image and schematic diagram of the system of asymmetrical electrodes.

When this system of strongly asymmetrical electrodes is connected to high DC voltage a well-defined flow of surrounding air is generated. This is caused by the ions generated in the vicinity of smaller electrode because of very high electric field strength around it, being accelerated towards the larger grounded electrode and colliding with neutral particles of surrounding air, thus creating the air flow. The exact mechanism and its description are analysed in detail in $[8,9]$.

In a preliminary test measuring with a CTA probe TESTO 425 close to the asymmetrical electrodes (connected to $14 \mathrm{kV}$ ) showed an oriented airflow with the velocities in the range of $0.2-0.7 \mathrm{~m} \cdot \mathrm{s}^{-1}$.

Following this a standard PIV measuring setup was built. It consisted of Nd:YAG green laser with wavelength $532 \mathrm{~nm}$ and high speed digital camera HiSense connected through a measurement card to a PC with Dantec Dynamics Studio software. The system of asymmetrical electrodes was placed into an insulating glass box to protect the surrounding sensitive electronic devices from overvoltage and also to shield the measuring space from any disturbing airflow. The dimensions of the box (400 x $400 \times 400 \mathrm{~mm})$ were large enough not to restrict the generated airflow, see Figure 2. Carbon particles with average size of $10 \mu \mathrm{m}$ were used as seeding particles. 


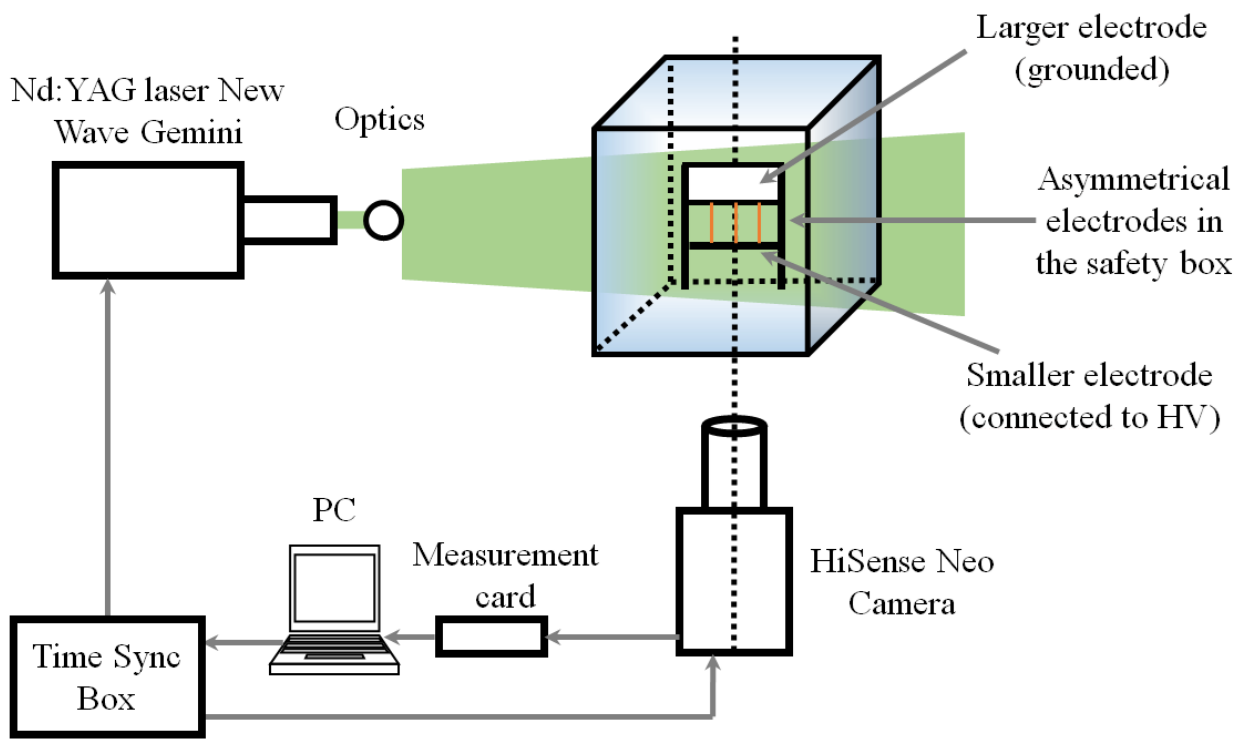

Fig. 2. Schematic diagram of measuring PIV setup.

Due to technical difficulties most measurements published so far $[10,11]$ were restricted to the area not exactly between the electrodes, where the largest part of the airflow generating phenomenon takes place. The main purpose of our experiment was to observe the flow directly between the two electrodes, because all theoretical descriptions of this phenomenon are derived for conditions valid specifically for this area.

The first technical difficulty that has to be mentioned is that because of asymmetrical electrodes geometry this area has the maximum value of electric field strength $(\sim 500 \mathrm{kV} / \mathrm{m})$. The second difficulty is the presence of the glass insulating rods, which are essential for the construction of this type of asymmetrical electrode system. The rods are blocking the optical access into the area between the electrodes and due to the need of using highly insulating materials (the most common being glass or plastics) there is a risk of unwanted strong reflections, which prevent the digital camera from recording the seeding particles correctly.

The presence of very high electric field strength was mitigated by using grounded Faraday cage around the digital camera and keeping safe distance between sensitive components and the experiment. The problem of laser reflections on the insulating rods was solved partially by coating their surface with special black lacquer. This paint does not contain metal particles or any other electrically conductive components, so it does not change electrical parameters of the rods. However the main solution of this problem was to choose an appropriate angle of the laser sheet with respect to the system of asymmetrical electrodes (in our case about 8 degrees from the axis), see Figure 3.

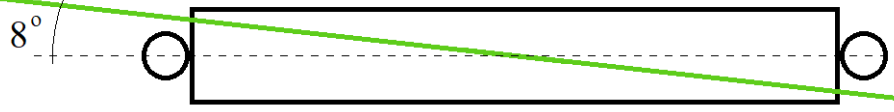

Laser sheet

Fig. 3. Schematic diagram of laser sheet angular relation with respect to the main axis of the electrodes (view from the top).

This view is close enough to the main axis to allow us to observe the area between the electrodes and it also prevents any undesired laser reflections. 


\section{Results}

The following PIV measurements were made for several different voltages. First state recorded was with no voltage showing the negligible airflow (in the range of $10^{-3} \mathrm{~m}_{\mathrm{s}} \mathrm{s}^{-1}$ ) velocities caused by the thermal movements of air in the insulating box, see Figure 4 .

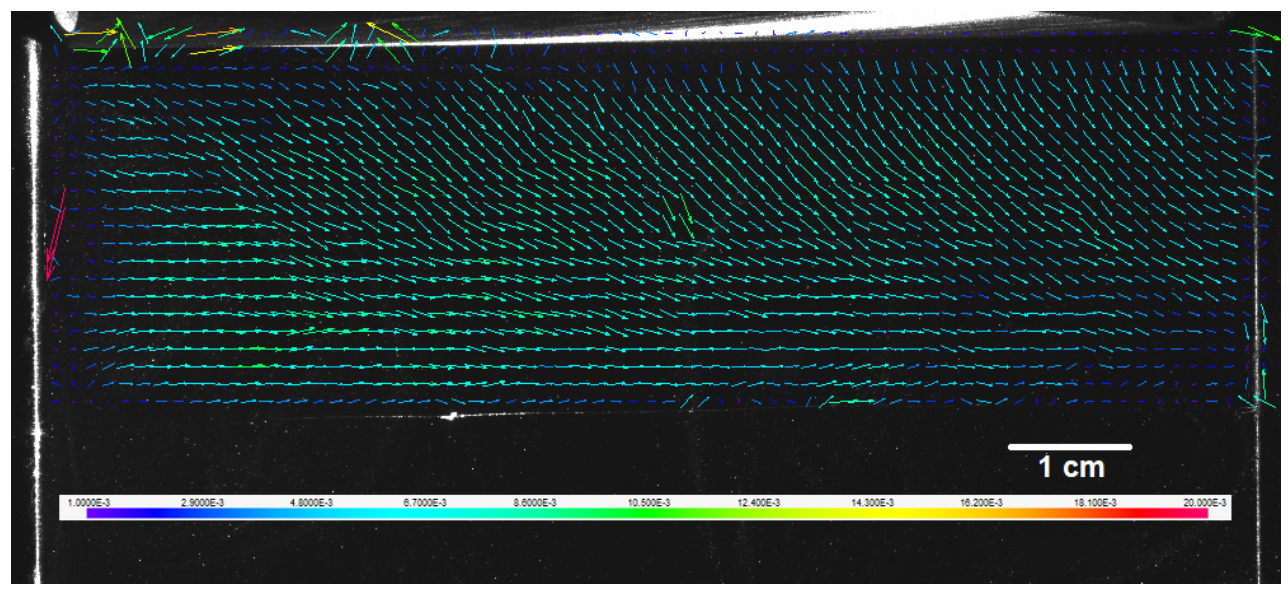

Fig. 4. PIV measurement of airflow velocities between the electrodes for zero voltage (and no electric current).

From the results in Figure 4 we can see that no significant airflow is present when no voltage is applied.

The following PIV measurements (see Figure 5-7) were made at several different voltages $(14 \mathrm{kV}, 16 \mathrm{kV}$ and $18 \mathrm{kV})$. These voltages were chosen since they were high enough for stable and measurable electric current to flow through the circuit, but low enough not to allow the creation of a spark discharge between the electrodes.

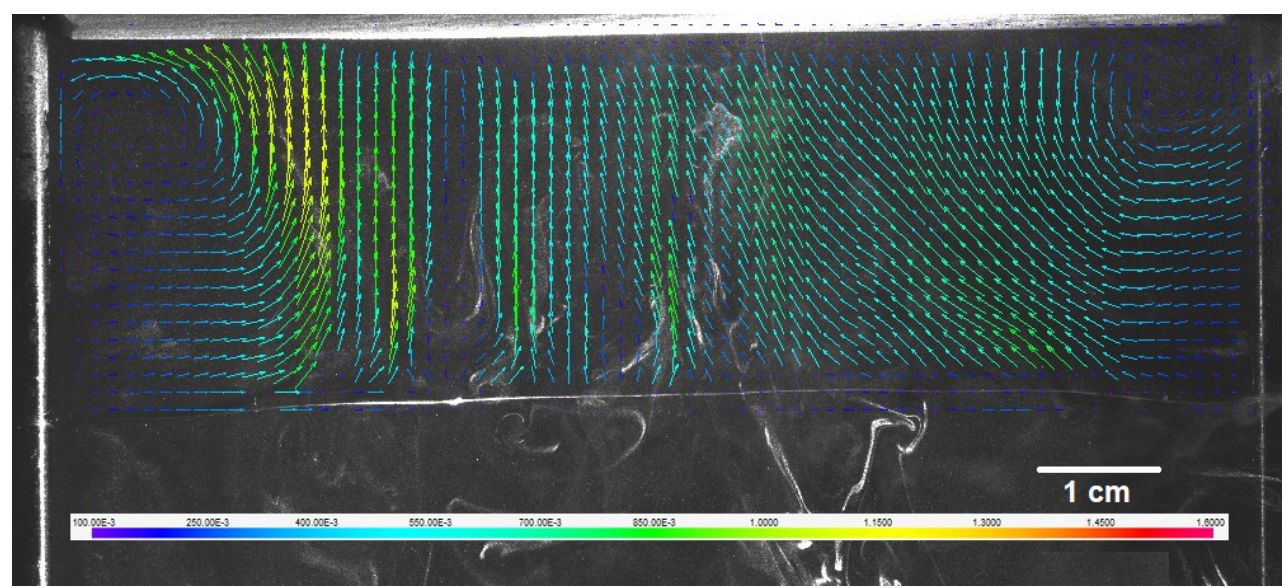

Fig. 5. PIV measurement of airflow velocities between the electrodes for the voltage $14 \mathrm{kV}$ (and electric current $16 \mu \mathrm{A})$. 


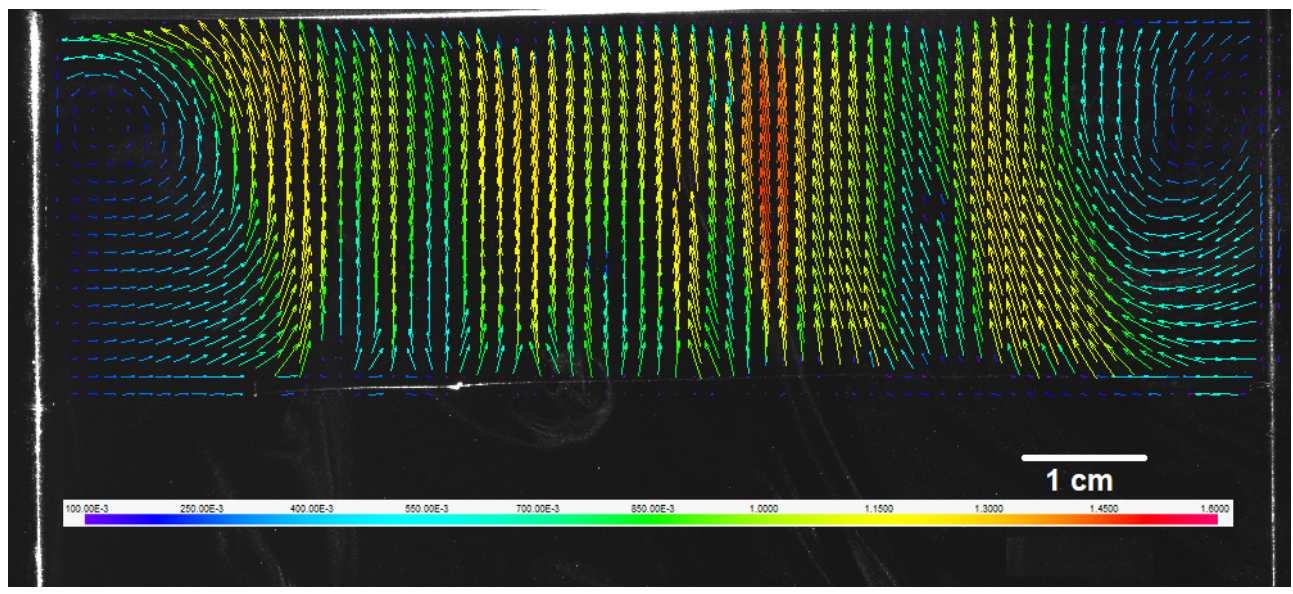

Fig. 6. PIV measurement of airflow velocities between the electrodes for the voltage $16 \mathrm{kV}$ (and electric current $33 \mu \mathrm{A})$.

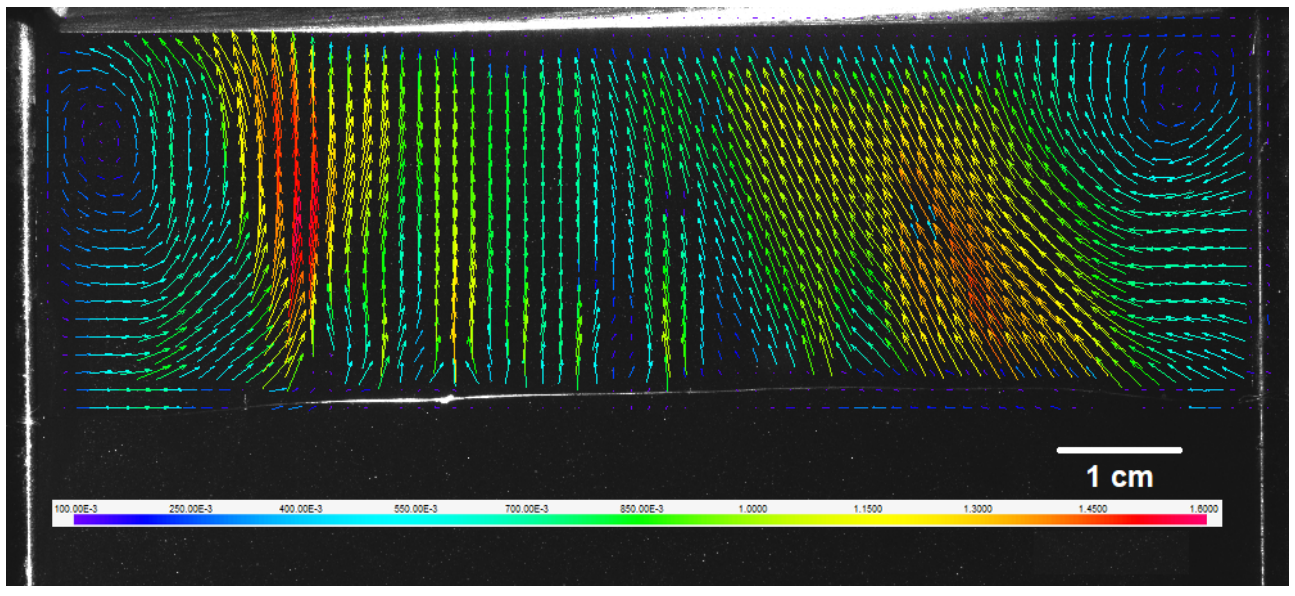

Fig. 7. PIV measurement of airflow velocities between the electrodes for the voltage $18 \mathrm{kV}$ (and electric current $58 \mu \mathrm{A})$.

The resulting PIV images in Figure 5-7 show a significant oriented airflow (in the range of $1-1.5 \mathrm{~m} . \mathrm{s}^{-1}$ ) is generated by applying high voltage on the system of asymmetrical electrodes. There is also an excellent correspondence between the published theory behind the airflow generating phenomenon [9] and our results, in that applying higher voltage (and thus higher electrical current flowing through the electrical circuit) generates higher airflow velocities.

\section{Discussion}

The results of our measurements show that PIV is definitely a viable method for measuring airflow generated by the high voltage phenomenon occurring on system of asymmetrical electrodes. Furthermore the results validate the theoretical description of observed airflow generating phenomenon. The most important conclusion, which can be drawn from the measurements, is the fact, that the airflow velocities are well in the range for possible practical applications $\left(v>1 \mathrm{~m} \cdot \mathrm{s}^{-1}\right)$, where macroscopic transport of gaseous media is 
desired. The main advantage of this airflow generating method is the absence of any moving parts, which implies much longer lifetime, durability and reduced noise production.

The first issue still remaining is the presence of high voltage in the range of tens of kilovolts, which however does not have to pose a serious difficulty, because the electric current is very low, only in the range of tens of microamperes. The second issue requiring further study is the possibility of chemical changes in the gaseous media due to the high electric field strength. This could result in generating ozone, nitrous oxides, free radicals, etc. and depending on their amount and stability this may turn into a problem for certain practical applications. However in other specific practical applications this may turn into an advantage - e.g. disinfection or decontamination.

Acknowledgement: The authors gratefully thank to the financial support of grant program PURE2020-6009 at Technical university of Liberec.

\section{References}

1. F. X. Canning, Asymmetrical Capacitors for Propulsion. NASA/CR-2004-213312 (2004)

2. G. Matsoukas, N. Ahmed, Investigation of Ionic Wind as a Means of Generating Propulsive Force. Int. Rev. of Aerospace Eng. 5, 2 (2012)

3. M. Raffel, C. E. Willert, F. Scarano, et al., Particle Image Velocimetry (Springer, New York, 2018)

4. Z. Zhengji, LDA Apllication Methods (Springer, Berlin, 2010)

5. C. Tropea, A. Yarin, J. F. Foss, Springer Handbook of Experimental Fluid Mechanics (Springer, Berlin, 2007)

6. S. Fialová, F. Pochylý, M. Kotek, D. Jasikova, Velocity profiles of fluid flow close to a hydrophobic surface. EPJ, 143 (2017)

7. R. Šulc, P. Ditl, I. For̆t, at al., The minimum record time for PIV measurement in a vessel agitated by a Rushton turbine. EPJ, 143 (2017)

8. J. Primas, M. Malík, V. Kopecký, M. Svoboda, Calculation and measurement of a neutral air flow velocity impacting a high voltage capacitor with asymmetrical electrodes. AIP Adv. 4, 1 (2014)

9. J. Primas, M. Malík, M. Svoboda, V. Kopecký, Biefeld-Brown effect Electrohydrodynamics phenomena on high voltage asymmetrical capacitor (Technical university of Liberec, Liberec, 2016)

10. W. A. Siswanto, K. Ngui, Performance of Triangular and Square Ionic Lifter Systems. Aust. J. of Bas. and Appl. Sc., 5 (2011)

11. M. Tajmar, T. Schreiber, Put Strong Limits on All Proposed Theories so far Assessing Electrostatic Propulsion. J. of Elst., 107 (2020) 